\title{
OLHARES SOBRE OS PROCESSOS FORMATIVOS EM ENFERMAGEM DE REABILITAÇÃO
}

\section{PERSPECTIVES ON TRAINING PROCESSES IN REHABILITATION NURSING}

\section{PERSPECTIVAS SOBRE LOS PROCESOS DE FORMACIÓN EN ENFERMERÍA DE REHABILITACIÓN}

\author{
José Miguel dos Santos Castro Padilha ${ }^{(1)}$, Maria Manuela Martins ${ }^{(2)}$, Narcisa Gonçalves ${ }^{(3)}$, Olga Ribeiro ${ }^{(4)}$, Carla Fernandes ${ }^{(5)}$, Bárbara
} Gomes $^{(6)}$

(1) Escola Superior de Enfermagem do Porto, Tech4edusim-CINTESIS, Porto, Portugal; (2) Escola Superior de Enfermagem do Porto (ESEP), Centro de Investigação em Tecnologias e Serviços de Saúde (CINTESIS), Porto, Portugal; (3) Escola Superior de Enfermagem do Porto, Porto, Portugal; (4) Escola Superior de Enfermagem do Porto (ESEP), Centro de Investigação em Tecnologias e Serviços de Saúde (CINTESIS), Porto, Portugal; (5) Escola Superior de Enfermagem do Porto (ESEP), Centro de Investigação em Tecnologias e Serviços de Saúde (CINTESIS), Porto, Portugal; (6) Escola Superior de Enfermagem do Porto (ESEP), Centro de Investigação em Tecnologias e Serviços de Saúde (CINTESIS), Porto, Portugal;

\section{Descritores}

Educação de Pós-Graduação em Enfermagem

Enfermagem

Enfermagem em reabilitação

\section{Descriptors}

Graduate Nursing Education Nursing

Rehabilitation Nursing

\section{Resumo}

Introdução: Nos últimos 50 anos, a formação pós-graduada no âmbito da especialização em Enfermagem de Reabilitação tem percorrido um trajeto de desenvolvimento técnico e disciplinar de forma a antecipar os desafios societais. Na última década tivemos um modelo de formação em uso que será substituído pelo modelo formativo publicado em março de 2021 pela Ordem dos Enfermeiros.

Objetivo: Analisar os resultados relativos à última década do processo formativo considerando as competências especificas dos enfermeiros especialistas em enfermagem de reabilitação.

Metodologia: Estudo de caso de natureza mista: quantitativa para os resultados do processo de aprendizagem e qualitativa para os processos organizativos. Na avaliação quantitativa, os scores médios, foram calculados a partir de uma escala de Likert de 5 pontos (onde 1 representa a pior opinião e 5 a melhor opinião). Os dados foram recolhidos a partir dos relatórios públicos de um curso de uma instituição de ensino de Enfermagem do Norte de Portugal.

Apresentação e interpretação dos resultados: Entre os anos letivos 2009/2010 e 2019/2020 concluíram o curso de Pós-Licenciatura de Especialização em Enfermagem de Reabilitação 289 estudantes e o de Mestrado em Enfermagem de Reabilitacão 64 estudantes. Realçam-se uma aproximacão das unidades curriculares às competências específicas dos Enfermeiros de Reabilitação e uma avaliação média, realizada pelos estudantes, de 4 para o componente interesse, 3,7 para o componente funcionamento e 4,2 para o componente desempenho dos professores.

Conclusão: Os resultados evidenciam que o curso de especialização e o mestrado em enfermagem de reabilitação são formações de sucesso, enquadradas nos articulados legislativos das competências dos especialistas em enfermagem de reabilitação, bem como realçam uma avaliação global média, dos processos de aprendizagem, muito positiva.

Abstract

Introduction: Over the last 50 years, postgraduate training within the scope of specialization in Rehabilitation Nursing has followed a path of technical and disciplinary development in order to anticipate societal challenges. In the last decade, we have had a training model in use that will be replaced by the training model published in March 2021 by the Portuguese Order of Nurses.

Objective: To analyze the results related to the last decade of the training process considering the specific competences of specialist nurses in rehabilitation nursing.

Methodology: Case study of mixed nature: quantitative for the results of the learning process and qualitative for the organizational processes. In the quantitative evaluation, the average scores were calculated from a 5-point Likert scale (where 1 represents the worst opinion and 5 the best opinion). Data were collected from public reports of specialization and master degree of a nursing education institution in the North of Portugal.

Presentation and interpretation of results: Between the academic years 2009/2010 and 2019/2020, 289 students completed the Specialization course in Rehabilitation Nursing, and 64 students completed the Master's Degree in Rehabilitation Nursing. There is evidence of an approximation of the curricular units to the specific competences of Rehabilitation Nurses and an average assessment performed by students of 4 for interest, 3.7 for functioning and 4.2 for teachers. 
Conclusion: The results show the specialization course in rehabilitation nursing as a successful training and framed in the legislative articulations of the competences of specialists in rehabilitation nursing, as well as a very positive global average assessment of the learning processes.

\section{Descriptores}

Educación de Postgrado en Enfermería

Enfermería

Enfermería de Rehabilitación

\section{Resumen}

Introducción: Durante los últimos 50 años, la formación de posgrado en el ámbito de la especialización en Enfermería de Rehabilitación ha seguido un camino de desarrollo técnico y disciplinar para anticipar los desafíos de la sociedad. En la última década, hemos tenido un modelo de formación en uso que será sustituido por el modelo de formación publicado en marzo de 2021 por la Ordem dos Enfermeiros.

Objetivo: Analizar los resultados relacionados con la última década del proceso de formación considerando las competencias específicas de los enfermeros especialistas en enfermería de rehabilitación.

Metodología: Estudio de caso de carácter mixto: cuantitativo para los resultados del proceso de aprendizaje y cualitativo para los procesos organizacionales. En la evaluación cuantitativa, las puntuaciones medias se calcularon a partir de una escala Likert de 5 puntos (donde 1 representa la peor opinión y 5 la mejor opinión). Los datos se obtuvieron de informes públicos de un curso en una institución de educación de enfermería en el norte de Portugal.

Presentación e interpretación de resultados: Entre los años académicos 2009/2010 y 2019/2020, 289 estudiantes completaron el curso de Especialización Post-Licenciatura en Enfermería de Rehabilitación y 64 estudiantes completaron el Máster en Enfermería de Rehabilitación. Se evidencia una aproximación de las unidades curriculares a las competencias específicas de las Enfermeras de Rehabilitación y una valoración media realizada por los alumnos de 4 por interés, 3,7 por funcionamiento y 4,2 por docente.

Conclusión: Los resultados muestran el curso de especialización y el Máster en enfermería Rehabilitación como una formación exitosa y enmarcada en las articulaciones legislativas de las competencias de los especialistas en enfermería de Rehabilitación, así como una valoración media global muy positiva de los procesos de aprendizaje.

\section{INTRODUÇÃO}

Em Portugal, a origem da enfermagem de reabilitação remonta à década de 60. Neste período a enfermeira Sales Luís assumiu um papel central na conceção, implementação e coordenação dos trabalhos que estiveram na origem do primeiro curso de enfermagem de reabilitação, cujo início foi a 18 de outubro de $1965^{(1-2)}$. Desde essa altura, e ao longo de mais de meio século, os enfermeiros de reabilitação têm vindo a consolidar o seu campo de ação e a verem reconhecida a relevância e contributo da enfermagem de reabilitação para a qualidade dos cuidados de saúde prestados aos Portugueses ${ }^{(1-3)}$.

A evolução do conhecimento, aliada à complexidade crescente dos cuidados de saúde, conduziram à necessidade de formação diferenciada e especializada na área dos cuidados de saúde e da Enfermagem. Nesse sentido, a formação especializada em Enfermagem assume um papel estratégico na qualificação de recursos humanos, com vista à otimização e racionalização dos cuidados de saúde, tendo por base a adequação do perfil de competências dos profissionais às exigências e desafios do sistema de saúde Português. A formação especializada na área de enfermagem de reabilitação assume particular relevẩncia dado que potencia a aquisição de conhecimentos e o desenvolvimento de competências essenciais às respostas aos principais desafios em saúde na contemporaneidade.

A formação académica na área específica de enfermagem de reabilitação, realizada em Portugal, tem um caráter distintivo pela regulação e formalização, enquanto área de especialidade ${ }^{(4)}$, não encontrando paralelo noutros países da Europa. No mundo, é encontrada em poucos países, apesar haver enfermeiros a trabalhar na área da reabilitação em muitos países ${ }^{(5)}$.

A origem da enfermagem de reabilitação no Mundo e em Portugal está intimamente relacionada com o elevado número de jovens com deficiências adquiridas em cenários de Guerra, como foram exemplos a segunda guerra Mundial, a guerra do Vietnam ou das antigas colónias Portuguesas ${ }^{(6)}$.

Nas últimas décadas do século XX e no início do século XXI temos assistido a alterações políticas, demográficas e à evolução dos sistemas de saúde, resultantes de novas configurações dos determinantes da saúde. O envelhecimento das populações; o aumento da prevalência de pessoas que vivem com doencas crónicas; a maior sobrevida a eventos de saúde agudos, a acidentes rodoviários e de trabalho, coloca desafios e gera novas oportunídades de desenvolvimento à área de especialidade em reabilitação, para torná-la mais significativa para as pessoas, famílias e para os sistemas de saúde.

No passado recente, os desafios à multiprofissionalidade e multidisciplinariedade das equipas de saúde, para desenvolverem e implementarem respostas mais efetivas aos problemas de saúde das pessoas, gerou resistências infundadas, quer internas quer externas à profissão, que tiveram impacto no âmbito da intervenção dos enfermeiros especialistas em Enfermagem de reabilitação (EEER). Mas, é clara a definição do enfermeiro especialista em Enfermagem de reabilitacão, como sendo aquele que cuida de pessoas com necessidades especiais, ao longo do ciclo de vida, em todos os contextos da prática de cuidados, capacita a pessoa com deficiência, limitação da atividade e ou restrição da participação para a reinserção e exercício da cidadania e que maximiza a funcionalidade desenvolvendo as capacidades da pessoa (4). Na atualidade, num contexto "quase pós-pandémico", a relevância e âmbito de ação do EEER encontra-se claramente explicitado nos resultados em saúde e satisfação das populações, no quadro do sistema de saúde Português.

Quando mergulhamos na reflexão sobre a reabilitação, facilmente identificamos a interação entre a conceção dos cuidados pelos EEER, com a perceção das pessoas portadoras de deficiência, quanto aos resultados alcançados em termos de funcionalidade, integração e participação social e, consequentemente, na melhoria global do bem-estar, sucesso individual e qualidade de vida (7). As pessoas portadoras de deficiência percecionam a reabilitação, e a ação dos EEER como essenciais para reeducação das funcões e estruturas corporais e/ou para a reeducação/reabilitação das atividades, nomeadamente de autocuidado, que no conjunto viabilizam a integração e plena participação social, através da reinserção no mercado de trabalho e na vida social.

Os desafios colocados à formação do EEER, na segunda década do século XXI, apontam para o desenvolvimento de competências para a facilitação e promoção de processos de transição e adaptação, centrados nas circunstâncias individuais e no ambiente envolvente das pessoas/família ${ }^{(8)}$. Os EEER têm uma intervenção central no desenvolvimento de competências nas pessoas com deficiência para a: 
autoajuda, autocuidado ${ }^{(9)}$, transição saudável ${ }^{(10)}$, gestão e tomada de decisão em saúde, tornando as pessoas/famílias em elementos ativos e motrizes do processo de reabilitação ${ }^{(10-12)}$. Contribuem, assim, para os resultados de saúde, para a racionalização de custos associados à diminuição dos períodos de hospitalização, para a redução do impacte da deficiência na participação, e para a melhoria da qualidade de vida ${ }^{(13)}$

Na segunda década do século XXI, é o momento de mudança do modelo de formação do EEER, e por isso torna-se relevante analisar e discutir o percurso da formação de forma a "iluminar o princípio" de um novo caminho a percorrer para a melhoria da qualidade formativa e consequentemente para tornar mais visível e significativa a ação dos EEER para os cidadãos.

$\mathrm{Na}$ análise das competências específicas dos EEER, em Portugal, em confronto com os quadros teóricos, as correntes filosóficas e os conceitos centrais da disciplina e profissão, identifica-se, claramente, uma componente da ação que transcende os processos de recuperação centrados nos compromissos dos processos e estruturas corporais. Nesta análise, a pessoa e a família emergem como objeto/alvo de intervenção do EEER, para a otimização dos processos de transição/adaptação à condição de saúde e aos desafios gerados pela alteração da funcionalidade, em consequência da deficiência, cuja finalidade é a minimização da limitação na participaçãoo e na integracão social, e a otimização da qualidade de vida. A própria Organizacão Mundial de Saúde tem vindo a alertar para a necessidade de os cuidados de saúde passarem a ser organizados de acordo com as necessidades e expectativas das pessoas e comunidades, mais do que centrados nas doenças. Estes factos reforçam os propósitos de repensarmos os processos formativos e avaliarmos os percursos realizados (14)

Assim, e tendo em consideração os desafios societais a Ordem dos Enfermeiros publicou em março de 2021 (15) o novo Programa formativo que integra o ciclo de estudos do curso de mestrado que visa o desenvolvimento de competências específicas do EEER.

A nossa instituição de ensino tem uma longa experiência de ensino especializado em enfermagem de reabilitacão. 0 primeiro curso teve início em 1985, tendo-se experienciado vários planos curriculares até à atualidade. Contudo, e apesar de pequenas alterações, o último plano curricular, foi implementado ao longo da última década na formação dos EEER. No referido plano curricular existiam dois cursos, o Curso de Pós-Licenciatura de Especializaç̃̃o em Enfermagem de Reabilitacão (CPLEER) composto por 60 ECTS (1 ano letivo) e o Curso de Mestrado em Enfermagem de Reabilitação (MER) composto por 120 ECTS (2 anos letivos). Ambos os cursos partilhavam as unidades curriculares que constituíam o primeiro ano (60 ECTS). O CPLEER e o $1^{\circ}$ ano do MER tinham por finalidade desenvolver competências específicas do EEER. No MER as unidades curriculares do $2^{\circ}$ ano tinham por finalidade desenvolver competências na área de investigação em Enfermagem de reabilitação, através de quatro unidades curriculares (Investigação em Enfermagem; Metodologias de análise qualitativa de dados; Metodologias de análise quantitativa de dados; Dissertação).

No momento atual, em que se pretende iniciar um novo programa formativo, que integre novos conteúdos, estratégias e contextos de aprendizagem dirigidos para o desenvolvimento de competências especificas e especializadas em enfermagem de reabilitação, torna-se relevante avaliar o percurso de ensino/ aprendizagem desenvolvido na última década, através da avaliação realizada pelos estudantes, que frequentaram os cursos de Pós-Licenciatura e Mestrado de Enfermagem de Reabilitação.

\section{METODOLOGIA}

Realizou-se um estudo de caso descritivo, retrospetivo, de métodos mistos, com o objetivo de analisar os resultados do processo formativo considerando as competências especificas dos enfermeiros especialistas em enfermagem de reabilitação.

O caso selecionado incluiu os modelos formativos associados ao curso de pós-licenciatura de especializacão em enfermagem de reabilitação (CPLEER) e mestrado de enfermagem de reabilitação (MER), realizados numa escola de Enfermagem pública do Norte do País. Estes cursos foram escolhidos porque pertencem a uma das primeiras escolas de enfermagem do país, com elevado legado e representação no âmbito da especialização em enfermagem de reabilitação. Para o efeito foi realizado um levantamento dos planos de estudos e resultados académicos no período compreendido entre os anos letivos 2009/2010 e 2019/2020. Os dados foram recolhidos a partir dos relatórios de avaliação anuais dos cursos que são do domínio público. Este percurso não carece da aprovação de comissão de ética dada a utilização de fontes públicas de dados.

\section{Análise de dados}

Realizou-se uma análise mista dos dados com base no relatório público anual de avaliação do curso do CPLEER e MER. A descritiva relativa ao número de estudantes inscritos; número de estudantes formados (CPLEER e MER); notas médias de curso das Unidades Curriculares; apreciação relativa ao interesse do estudante pela Unidade Curricular; apreciação relativa ao funcionamento da Unidade Curricular; apreciação relativa aos docentes da Unidade Curricular. A apreciação dos estudantes foi realizada através de uma escala de Likert de 5 pontos (onde 1 representa a pior opinião e 5 a melhor opinião). A análise qualitativa englobou a análise das temáticas da Unidade Curricular de dissertação do MER recorremos à análise de conteúdo ${ }^{(16)}$, com um modelo de análise à priori, no qual as categorias foram organizadas em torno das unidades curriculares especificas dos cursos e relativas ao $1^{\circ}$ ano.

\section{RESULTADOS}

Desde o ano letivo 2009/2010, nesta instituição de ensino, foram atribuídos diplomas de estudos especializados ou grau de mestre em Enfermagem de Reabilitacão a 353 Enfermeiros. Entre os anos letivos 2009/2010 e 2019/2020 concluiram o CPLEER 289 estudantes e 64 estudantes concluíram o MER.

Todos os dados que se apresentam estão agrupados e são relativos aos anos letivos compreendidos entre 2009/2010 e 2019/2020.

A idade média dos enfermeiros que concluíram a formação foi de 32 anos $(S D \pm 6,2$; Máx.=57; Mín.=24), não existindo diferenças com significado estatístico entre os estudantes que concluíram o curso de Pós-licenciatura ou o curso de Mestrado.

Relativamente à média da nota de conclusão do curso existem diferenças com significado estatístico entre os dois grupos de estudantes, tendo os estudantes do mestrado concluído com média mais alta 16,7 $(\mathrm{SD} \pm 0,9 ;$ Máx. $=18,9 ;$ Mín. $=13,7)\left(t_{351}=-8,165 ; p<0,001 ; \mathrm{d}=1.1\right)$, comparativamente aos estudantes do CPLEER com média 15,7 (SD=0,9; Máx.=17,9; Mín.=11,5).

Relativamente aos resultados nas unidades curriculares (UC's) do $1^{\circ}$ ano podemos analisar na tabela 1 as médias globais da classificação final dos estudantes, por curso. 
Tabela 1 - Médias globais/ curso da classificação final dos estudantes das unidades curriculares do $1^{\circ}$ ano

\begin{tabular}{|c|c|c|c|}
\hline \multirow{2}{*}{ Unidade curricular } & \multicolumn{3}{|c|}{ Média da classificação final por UC/ $1^{\circ}$ ano } \\
\hline & Global UC & CPLEER & MER \\
\hline Epistemologia de enfermagem & 15,2 & 15,4 & 15,0 \\
\hline Bioética & 14,1 & 14,2 & 14,0 \\
\hline Supervisão clínica & 15,6 & 15,7 & 15,5 \\
\hline Prática baseada na evidência & 16,6 & 16,8 & 16,3 \\
\hline Enfermagem de Reabilitação & 15,2 & 15,4 & 14,9 \\
\hline Famílias e a Pessoa com Deficiência & 14,4 & 14,5 & 14,4 \\
\hline Cinesiologia Humana & 14,2 & 14,6 & 13,8 \\
\hline Integração e Cidadania & 15,2 & 15,2 & 15,2 \\
\hline A pessoa afeções ortotraumatológicas e conjuntivas & 16,2 & 16,4 & 16,0 \\
\hline A pessoa com afeções cardio respiratórias & 15,8 & 15,9 & 15,6 \\
\hline A pessoa com afeções Neurológicas & 16,2 & 16,3 & 16,0 \\
\hline Terapias complementares e reabilitação & 14,4 & 14,5 & 14,2 \\
\hline Atividade física e desenvolvimento humano & 16,3 & 16,4 & 16,1 \\
\hline Reabilitação Gerontogeriátrica & 16,4 & 16,2 & 16,7 \\
\hline
\end{tabular}

Na tabela 2 podemos analisar a média das classificações finais, relativas aos estudantes do MER, nas UC's do $2^{\circ}$ ano.

Tabela 2 - Média das classificações finais dos estudantes do MER nas UC's do $2^{\circ}$ ano.

\begin{tabular}{|l|c|}
\hline Unidades curriculares & Média da classificação final por UC/2 ${ }^{\circ}$ ano \\
\hline Investigação em Enfermagem & 13,6 \\
\hline Metodologias de análise qualitativa de dados & 15,5 \\
\hline Metodologias de análise quantitativa de dados & 15,4 \\
\hline Dissertação & 17,7 \\
\hline
\end{tabular}

No final do semestre de funcionamento de cada unidade curricular, os estudantes foram convidados a avaliar as UCs relativamente ao interesse, ao funcionamento e ao desempenho dos professores. A avaliação foi feita em formato eletrónico, voluntária, utilizando uma escala tipo Likert de 5 pontos, onde 1 representa a pior opinião e 5 a melhor opinião.

Na tabela $n .^{\circ} 3$, podemos analisar os scores globais de interesse, funcionamento e desempenho dos professores de cada uma das unidades curriculares do CPLEER e MER. Os dados relativos às UCS do $1^{\circ}$ ano são apresentados de forma agregada dado não existirem diferenças com significado estatístico, relativas a cada uma das dimensões em análise, entre os cursos.

Tabela 3 - Scores globais atribuídos pelos estudantes ao interesse, funcionamento e desempenho dos professores de cada uma das UCS do CPLEER e MER.

\begin{tabular}{|l|c|c|c|}
\hline \multirow{2}{*}{ Unidades curriculares } & \multicolumn{2}{l}{ Score global } & \multicolumn{2}{l|}{} \\
\cline { 2 - 4 } & Interesse & Funcionamento & Professores \\
\hline Epistemologia de enfermagem & 4,0 & 3,6 & 3,2 \\
\hline Ética de Enfermagem & 3,9 & 3,0 & 4,3 \\
\hline Introdução à Supervisão Clínica em Enfermagem & 4,0 & 3,6 & 4,1 \\
\hline Prática baseada na evidência & 3,9 & 3,6 & 4,1 \\
\hline Enfermagem de Reabilitação & 4,1 & 3,8 & 4,2 \\
\hline Famílias e a Pessoa com Deficiência & 4,0 & 3,5 & 4,1 \\
\hline Cinesiologia Humana & 4,1 & 3,8 & 3,8 \\
\hline A pessoa com afeções cardio respiratórias & 3,9 & 3,3 & 4,0 \\
\hline Integração e Cidadania & 4,2 & 3,9 & 4,4 \\
\hline A pessoa com afeções Neurológicas & 4,2 & 3,8 & 4,1 \\
\hline A pessoa afeções ortotraumatológicas e conjuntivas & 4,3 & 3,7 & 3,3 \\
\hline Terapias complementares e reabilitação & 3,5 & 2,8 & 4,7 \\
\hline Reabilitação Gerontogeriátrica & 4,0 & 4,3 & 4,6 \\
\hline Atividade física e desenvolvimento humano & 4,2 & 4,1 & 3,9 \\
\hline Investigação em Enfermagem* & 3,4 & 3,8 & 4,1 \\
\hline Metodologias de análise qualitativa de dados* & 3,6 & 4,0 & 4,1 \\
\hline Metodologias de análise quantitativa de dados* & 3,7 & 3,9 & 4,8 \\
\hline Investigação em Enfermagem* & 4,2 & 4,0 & - \\
\hline Dissertação & & - & \\
\hline
\end{tabular}


No final do ano letivo os estudantes foram convidados a avaliar os cursos, de um modo global, relativamente ao interesse, ao funcionamento e desempenho dos professores numa escala de Likert de 5 pontos, onde 1 representa a pior opinião e 5 a melhor opinião.

$\mathrm{Na}$ tabela $\mathrm{n} .{ }^{\circ} 4$ podemos analisar os scores globais atribuídos pelos estudantes ao interesse, funcionamento e professores dos cursos CPLEER e MER.

Tabela 4 - Scores médios globais atribuídos pelos estudantes ao interesse, funcionamento e desempenho dos professores dos cursos CPLEER e MER

\begin{tabular}{|l|c|c|c|}
\hline Curso & Interesse & Funcionamento & Professores \\
\hline CPLEER & 4,1 & 3,6 & 4,1 \\
\hline MER & 3,9 & 3,7 & 4,2 \\
\hline GLOBAL & 4 & 3,7 & 4,2 \\
\hline
\end{tabular}

No período em análise concluíram a unidade curricular de dissertação com a defesa pública 64 estudantes. Na análise de conteúdo às temáticas e agrupando-as, em função das UC especificas do $1^{\circ}$ ano, podemos verificar a distribuição de temáticas de dissertação na tabela n. ${ }^{\circ} 5$.

Tabela 5 - Distribuição das temáticas de dissertação em Enfermagem Reabilitação

\begin{tabular}{|l|c|}
\hline Unidade curricular & Média \\
\hline Enfermagem de Reabilitação & $39,7 \%$ \\
\hline Famílias e a Pessoa com Deficiência & $10,3 \%$ \\
\hline Integração e Cidadania & $13,8 \%$ \\
\hline A pessoa afeções ortotraumatológicas e conjuntivas & $3,4 \%$ \\
\hline A pessoa com afeções cardiorrespiratórias & $20,7 \%$ \\
\hline A pessoa com afeções Neurológicas & $12,1 \%$ \\
\hline
\end{tabular}

\section{DISCUSSÃO}

No âmbito da saúde, a formação pós-graduada em áreas de especialização tem como principal objetivo promover o desenvolvimento de competências direcionadas para uma área específica de intervenção, tendo como finalidade dar resposta às necessidades de cuidados que vão emergindo ${ }^{(17)}$.

No contexto português, na área disciplinar de enfermagem, apesar do investimento efetuado ao longo das últimas décadas em cursos de especialização, as mudanças sociodemográficas e a evolução técnico-científica, bem como a existência de novas orientações quanto aos programas formativos das especializações em enfermagem, e particularmente em enfermagem de reabilitação, reforcaram a necessidade de reflexão sobre o percurso que nos conduziu à atualidade. Além disso, para uma instituição de ensino, que sempre se destacou pela qualidade e diferenciação dos processos de ensino/aprendizagem, torna-se inevitável analisar o caminho percorrido, agora que se encerra um ciclo, de forma a garantir que as aprendizagens emergentes orientarão as decisões futuras, assegurando desta forma que um legado de rigor, qualidade e reconhecimento externo, tenha continuidade no futuro.

O envolvimento dos enfermeiros nos cursos de pós-licenciatura de especialização e mestrado em enfermagem de reabilitação, têm sido associados, essencialmente na última década, a percursos individuais desafiantes, pelo facto de serem realizados em regime de trabalhadorestudante. Contudo, apesar da dificuldade de gestão do tempo profissional, de estudante, familiar e pessoal, a média global dos processos de aprendizagem foi, neste período, bastante positiva. Não obstante, da métrica observada ou da qualidade formativa percecionada pelos EEER, pelos pares e instituições de cuidados, estes percursos, nas palavras dos envolvidos, carecem de um enquadramento e suporte formal mais facilitadores. As médias globais refletem, o envolvimento dos enfermeiros - estudantes, mas também das instituições de ensino e dos seus professores para encontrarem as soluções pedagógicas promotoras do desenvolvimento de competências e facilitadoras dos processos de aprendizagem.

Nos estudantes que concluíram o MER, observamos que a média global foi 1 valor superior à média dos estudantes que terminaram o curso CPLEER, facto explicado pelo peso relativo da nota da unidade curricular do $2^{\circ}$ ano "Dissertação", na média final do MER.

Nas unidades curriculares do primeiro semestre, transversais ao CPLEER e ao MER, as classificações globais variaram entre 14,1 e 16,4 valores.

Atendendo a que no modelo formativo atualmente em vigor, tendencialmente organizado em torno de alguns processos patológicos, o desenvolvimento de competências específicas na área de enfermagem de reabilitação ${ }^{(4)}$ está fortemente relacionado com os conteúdos lecionados nas unidades curriculares "A pessoa com afeções cardiorrespiratórias", "A pessoa com afeções neurológicas" e "A pessoa afeções ortotraumatológicas e conjuntivas", os resultados obtidos incitaram a nossa reflexão. Efetivamente, as classificaçỗes globais dessas três UC, que variaram entre 15,8 e 16,2, além de traduzirem o investimento dos estudantes, revelam indiretamente a importância que atribuem às principais áreas clínicas da enfermagem de reabilitação.

Da pouca variabilidade dos resultados da aprendizagem nessas três unidades curriculares, e perspetivando um novo modelo formativo mais centrado nas respostas humanas aos processos de transição ${ }^{(18)}$, depreende-se a necessidade de no futuro se investir em estratégias pedagógicas inovadoras que potenciem o envolvimento dos estudantes, a translação do conhecimento e a transferibilidade das competências para a prática clínica. E neste contexto, um dos principais objetivos será disponibilizar aos estudantes as ferramentas que lhes permitam atingir gradualmente e de forma sustentada, diferentes níveis de proficiência na área da enfermagem de reabilitação (7). 0 investimento desigual nas áreas clínicas específicas, poderá defraudar expectativas e gerar desmotivação. De facto, ainda que os EEER reconheçam que na sua prática clínica nem sempre é possível dar resposta a todas as necessidades em cuidados de reabilitação que as pessoas apresentam (19), na formação pós-graduada de especialização nenhuma das áreas poderá ser descurada.

No que se refere à avaliação do interesse, funcionamento e desempenho dos professores das UC, o componente com média inferior foi o funcionamento. A distribuicão das aulas em vários dias da semana, a par da percecão dos estudantes face à elevada carga de trabalho, poderá justificar esse resultado, nomeadamente no primeiro semestre, que efetivamente concentra um maior número de ƯCs. Este facto, está intimamente relacionado com a inexistência de uma política integrada e transversal de formação pós-graduada em Enfermagem, em 
Portugal que permita aos enfermeiros conciliarem a formação pós-graduada com a atividade profissional, de forma similar ao que acontece em outras áreas de formação pós-graduada na área da saúde.

Nos últimos anos, na sequência do reconhecimento de que a investigação é fundamental para o desenvolvimento do conhecimento na área da enfermagem de reabilitação e da qualidade e segurança dos cuidados de enfermagem de reabilitação, os enfermeiros aceitaram o desafio de enveredar por um percurso formativo que além do título profissional de EEER lhes permitiu obter o grau académico de mestre (20). Essa opção permitiu o desenvolvimento de investigação em áreas distintas da enfermagem de reabilitação.

Considerando que em Portugal, o desenvolvimento da investigação nas áreas de especialização em enfermagem, e especificamente na reabilitação, é recente, mostra-se compreensível o investimento inicial em temáticas associadas à UC de "Enfermagem de Reabilitação" $(39,7 \%)$, muito centradas nas competências dos EEER, nos referenciais teóricos orientadores do exercício profissional e no processo de tomada de decisão destes especialistas.

Da análise efetuada, sobressai ainda que as temáticas incluídas nas unidades curriculares "A pessoa com afeções ortotraumatológicas e conjuntivas" e "Famílias e a Pessoa com Deficiência" foram as menos encontradas nas dissertações. Estes dados, permitem-nos perceber e definir áreas de atenção prioritárias para a investigação no futuro próximo, desenvolvendo projetos mais abrangentes onde os estudantes possam ancorar a sua investigação.

Este estudo tem por limitação circunscrever-se apenas numa instituição de ensino superior. Contudo, é um contributo para a reflexão do que podemos melhorar e servirá como ponto de comparação com o novo plano de estudos.

\section{CONCLUSÃO}

Este estudo apresenta-nos os resultados do processo formativo realizados nos últimos 10 anos nos cursos do CPLEER e MER de uma instituição de ensino do Norte de Portugal. Os resultados evidenciam o Curso de Especialização em Enfermagem de Reabilitacão (CPLEER) e o Mestrado de Enfermagem de Reabilitação (MER), como formações de sucesso e enquadradas nos articulados legislativos e nas competências especificas dos enfermeiros especialistas em enfermagem de reabilitação, bem como mostram uma avaliação global média dos processos de aprendizagem muito positiva. O MER mostra o percurso de desenvolvido na investigação em enfermagem de reabilitação, na última década, e as áreas da prática clínica que suscitaram mais interesse.

A efetividade dos processos formativos, na área de Enfermagem de Reabilitação, resultou de múltiplos fatores, nomeadamente da interação com os contextos da prática clínica. Neste sentido, estes cursos assumiram-se, também, como impulsionadores de novas práticas clínicas, que consequentemente influenciaram, também, os percursos formativos na academia, alicerçados, sempre, numa dialética interativa subjacente ao desenvolvimento epistemológico.

Os resultados apresentados, não podem, nem pretendem representar todo o processo desenvolvido ao longo de uma década por estudantes, docentes e pela instituição. Pretendem apenas, sustentar a discussão e orientar as decisões futuras na formação pós-graduada de Especialização em Enfermagem de Reabilitação.

\section{DIVULGAÇõES ÉTICAS}

\section{Contribuição do(s) autor(es):}

Conceptualização: MP; MM.

Metodologia: MP; MM.

Validação: MP; MM; OR; CF; NG; BG.

Análise formal: MP; MM; OR; CF; NG; BG;

Investigação: MP; MM;

Tratamento de dados: MP; MM;

Preparação do rascunho original: MP; MM; OR; CF; NG; BG;

Redação e edição: MP; MM; OR; CF; NG; BG;

Revisão: MP; MM; OR; CF; NG; BG;

Todos os autores leram e concordaram com a versão publicada do manuscrito.

\section{Financiamento:}

Este trabalho não recebeu nenhuma contribuição financeira ou bolsa.

\section{Comissão de Ética:}

Estudo utiliza fontes públicas.

\section{Declaração de consentimento informado:}

Não aplicável.

\section{Agradecimentos:}

Os autores agradecem e reconhecem o envolvimento e qualidade da colaboração de todos os Professores, enfermeiros especialistas em Enfermagem de Reabilitação, Órgãos da instituição de ensino e instituições prestadoras de cuidados que contribuíram para o sucesso dos percursos formativos em análise neste artigo.

Conflitos de interesse: 
Os autores não declaram nenhum conflito de interesses.

\section{Proveniência e revisão por pares:}

Não comissionado; revisto externamente por pares.

\section{Referências}

1. Fernandes CS, Gomes JA, Magalhães BM, Lima AMN. Produção de conhecimento em enfermagem de reabilitação portuguesa - scoping review. J Health NPEPS [serial on the Internet]. 2019 [cited 2021 May 21]; 4(1):282-301. Doi: 10.30681/252610103378

2. Ordem dos Enfermeiros. Padrão Documental dos Cuidados de Enfermagem da Especialidade de Enfermagem de Reabilitação [serial on the Internet]. Lisboa: Ordem dos Available https://www.ordemenfermeiros.pt/arquivo/colegios/Documents/2015/MCEER_Assembleia/PadraoDocumental_EER.pdf

3. Spasser MA, Weismantel A. Mapping the literature of rehabilitation nursing. J Med Libr Assoc [serial on the Internet]. 2006 [cited 2021 May 20];94(2 Suppl):e137-42. Retrieved from: https://www.ncbi.nlm.nih.gov/pmc/articles/PMC1463035/pdf/i1536-5050-094-02S-0137.pdf

4. Ordem dos Enfermeiros. Regulamento das Competências Específicas do Enfermeiro Especialista em Enfermagem de Reabilitação [serial on the Internet]. Lisboa: Ordem dos Enfermeiros; 2019 [cited 2021 May 22]. Available from: https: / /dre.pt/application/conteudo/122216893

5. Schoeller S, Martins MM, Ribeiro I, Lima DKS, Padilha MI, Gomes BP. Breve panorama mundial da enfermagem de reabilitação. Rev Port Enf Reab [serial on the Internet]. 2018 [cited 2021 May 21] ; 1(1):6-12. Doi: 10.33194/rper.2018.v1.n1.01.4388

6. St-Germain D. The rehabilitation nurse then and now: From technical support to human potential catalyst by Caring- Disability Creation Process Model in an interprofessional team. J Nurs Educ Pract [serial on the Internet]. 2014 [cited 2021 May 28]; 4(7):54-61. Doi: 10.5430/jnep.v4n7p54

7. Vaughn S, Mauk KL, Jacelon CS, Larsen PD, Rye J, Wintersgill W, et al. The Competency Model for Professional Rehabilitation Nursing. Rehabil Nurs [serial on the Internet]. 2016 [cited 2021 Jun 05];41(1):33-44. Doi: 10.1002/rnj.225

8. World Health Organization. World report on disability 2011 [serial on the Internet]. Geneva: World Health Organization; 2011. Available from: https://www.who.int/disabilities/world_report/2011/report.pdf

9. Orem D. Modelo de Orem, Conceptos de enfermería en la práctica. Barcelona: Ediciones Cienti?ficas y Te?cnicas; 1993.

10. Meleis Al. Theoretical nursing: development and progress. 5th ed. Philadelphia: Lippincott; 2012

11. Llewellyn G, Bundy A, Mayes R, McConnell D, Emerson E, Brentnall J. Development and psychometric properties of the family life interview. J Appl Res Intellect Disabil [serial on the Internet]. 2010 [cited 2021 May 22]; 23(1):52-62. Doi: 10.1111/j.1468-3148.2009.00545.x

12. Mola E, Bonis JA, Giancane R. Integrating patient empowerment as an essential characteristic of the discipline of general practice/family medicine. Eur J Gen Pract [serial on the Internet]. 2008 [cited 2021 May 22];14(2):89-94. Doi: 10.1080/13814780802423463.

13. Davies EJ, Moxham T, Rees K, Singh S, Coats AJ, Ebrahim S, et al. Exercise based rehabilitation for heart failure. Cochrane Database Syst Ver [serial on the Internet]. 2010 [cited 2021 Jun 02];(4):CD003331. Doi: 10.1002/14651858.CD003331

14. World Health Organization. WHO global strategy on integrated people-centred health services $2016-2026$ [serial on the Internet].

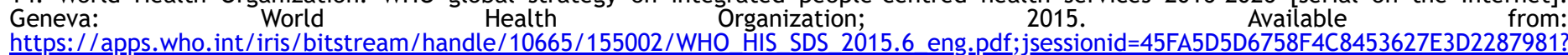
sequence $=1$

15. Ordem dos Enfermeiros. Percurso e Programa Formativo para a Especialidade de Enfermagem de Reabilitação [serial on the Internet]. Lisboa: Ordem dos Enfermeiros; 2014 [cited 2021 Jun https://www.ordemenfermeiros.pt/arquivo/colegios/Documents/2015/MCEER Assembleia/Percurso Programa Formativo EER.pdf

16. Bardin L. Análise de Conteúdo. Coimbra: Edições 70; 2018.

17. Silva RM, Luz MDA, Fernandes JD, Silva LS, Cordeiro ALA, Mota LDS. Becoming a specialist: Portuguese nurses' expectations after completing the specialization program. Rev Enf Ref [serial on the Internet]. 2018 [cited 2021 Jun 06];4(16):147-154. Doi: 10.12707/RIV17076

18. Silva R, Carvalho A, Rebelo L, Pinho N, Barbosa L, Araújo T, et al. Contributos do Referencial Teórico de Afaf Meleis para a Enfermagem de Reabilitação. Revista Investigação em Enfermagem [serial on the Internet]. 2019 [cited 2021 Jun 05]; (26):35-44. Available from: http: / / www. sinaisvitais.pt/images/stories/Rie/RIE26 s2.pdf

19. Prazeres VMP, Ribeiro CD, Marques GFS. Decision making for nursing rehabilitation in intensive care: foci of care. Rev Rene [serial on the Internet]. 2021 [cited 2021 Jun 04];22:e61706. Doi: 10.15253/2175-6783.20212261706

20. Portugal. Aviso n. ${ }^{\circ}$ 3915/2021, de 03 de março de 2021. Programa formativo que integra o ciclo de estudos do curso de mestrado que visa o desenvolvimento de competências específicas do enfermeiro especialista em Enfermagem de Reabilitação. 03 de março de 2021 . Available from: https: / / dre.pt/application/conteudo/158724032

Este trabalho encontra-se publicado com a Licença Internacional Creative Commons Atribuição-NãoComercial-SemDerivações 4.0.

Direitos de Autor (c) 2021 Revista Portuguesa de Enfermagem de Reabilitação 